Girish P. Joshi MB BS MD FFARCSI

\title{
Anesthesia for laparoscopic surgery
}

$\mathrm{T}$

HE development of minimally invasive surgery has revolutionized surgical procedures and in the process has influenced the practice of anesthesiology. The advantages of laparoscopic surgery include reduced overall adverse events, shorter hospital stay, and rapid return to normal activities. The success in healthy adult patients has led to the introduction of more extensive endoscopic procedures in older and sicker patients, as well as in pregnant and pediatric patients. Laparoscopic procedures that are increasing acceptance include adrenalectomy for pheochromocytoma, Nissen fundoplication for gastroesophageal reflux disease, gastric bypass for obesity, and nephrectomy (radical or donor). In addition, an increasing number of these procedures are being performed on an outpatient basis. ${ }^{1}$

Despite the potential advantages, laparoscopic procedures are associated with physiological changes, which present the anesthesia practitioner with many challenges. ${ }^{2}$ In addition, these procedures are associated with potential life-threatening complications that are usually not encountered with the traditional open procedure. ${ }^{3}$ Thus, there is a need to modify the anesthetic technique to allow these novel surgical procedures to be performed safely.

\section{Physiological changes during laparoscopy}

The cardiopulmonary changes during laparoscopy are complex and depend on the interaction of the patient's preexisting cardiopulmonary status, the anesthetic technique, and several surgical factors including intra-abdominal pressure (IAP), carbon dioxide $\left(\mathrm{CO}_{2}\right)$ absorption, patient position and duration of the surgical procedure. ${ }^{2}$ Although physiological changes are well tolerated by most healthy patients, they could have adverse consequences in the elderly and patients with limited cardiopulmonary reserve. ${ }^{2,3}$

\section{Cardiovascular changes}

Most investigations assessing the cardiovascular changes of laparoscopy report an increase in systemic vascular resistance, mean arterial pressure, and cardiac filling pres- sures. ${ }^{2}$ Changes in cardiac index and heart rate appear to be minimal in healthy patients. However, the hemodynamic effects in patients with preexisting disease can be more severe and there is a risk of sudden deterioration. ${ }^{4}$

The increase in systemic vascular resistance may be due to increased sympathetic output from $\mathrm{CO}_{2}$ absorption and a neuroendocrine response to pneumoperitoneum. The increase in systemic vascular resistance may increase the myocardial wall tension and thus, increase the myocardial oxygen demand. Compression of the abdominal organs (e.g., liver and spleen) as a result of increased IAP and increased sympathetic output may be one of the causes of increased cardiac filling pressures. ${ }^{5}$ In addition, the cardiac filling pressures may reflect the increased intrathoracic pressures caused by pneumoperitoneum.

Induction of pneumoperitoneum in the supine position (rather than head-down position) appears to reduce the alterations in cardiovascular function. ${ }^{5}$ Furthermore, the cardiopulmonary effects are proportional to the magnitude of IAP attained during laparoscopy with the significant changes occurring at pressures greater than $12 \mathrm{mmHg} .{ }^{6}$ Minimizing IAP should decrease the risk of potentially significant physiologic changes. ${ }^{7}$ In addition, hemodynamic derangement is also related to the surgical procedure. For example, surgical disruption of the esophageal hiatus during laparoscopic Nissen fundoplication may increase mediastinal and pleural pressures resulting in a significant reduction in cardiac output. ${ }^{8}$

Numerous regional circulatory changes also occur during laparoscopy including increased cerebral blood flow and intracranial pressure, decreased total hepatic blood flow, reduced bowel circulation resulting in decreased gastric intramucosal $\mathrm{pH}$ (suggesting reduced gut perfusion), reduction in renal blood flow and urine output, and decreased femoral vein blood flow which may increase the risk of deep vein thrombosis. ${ }^{2}$ Of importance, the changes in regional circulation may have clinical implications in critically ill patients undergoing extensive laparoscopic procedures requiring prolonged pneumoperitoneum.

From the Department of Anesthesiology and Pain Management, University of Texas Southwestern Medical Center, Dallas, Texas, USA.

Address correspondence to: Dr. Girish P. Joshi, Department of Anesthesiology and Pain Management, University of Texas Southwestern Medical Center, 5323 Harry Hines Blvd., Dallas, Texas, USA. Phone: 214-590-7259; Fax: 214-590-6945;

E-mail: girish.joshi@utsouthwestern.edu 


\section{Pulmonary changes}

The increased IAP displaces the diaphragm upward and decreases functional residual capacity and total lung compliance, resulting in basal atelectasis and increased airway pressures. ${ }^{2}$ The increase in minute ventilation required to maintain normocarbia further increases peak airway pressures. These pulmonary changes are compounded with those caused by changes in position. Furthermore, these changes may be exaggerated in obese patients and in those with respiratory dysfunction.

\section{Anesthetic management}

An optimal anesthetic technique should provide excellent intraoperative conditions while ensuring rapid recovery, low incidence of adverse effects, and early return to daily activities. ${ }^{9}$ General anesthesia with muscle paralysis and tracheal intubation remains the preferred technique for most laparoscopic procedures. Muscle paralysis reduces the IAP needed for the same degree of abdominal distention. Maintenance of anesthesia with inhaled anesthetic (e.g., desflurane and sevoflurane) allows for rapid recovery as compared with infusion of propofol (i.e., total $i v$ anesthesia). ${ }^{10}$ Furthermore, use of bispectral index monitoring to titrate short-acting anesthetics has also been shown to expedite recovery. ${ }^{11}$ Once adequate depth of hypnosis is achieved, use of vasoactive drugs such as esmolol or labetalol may be more appropriate to treat hypertension caused by pneumoperitoneum. On the other hand, use of hypnotic-sedatives or opioids to treat pneumoperitoneum-related hypertension might delay emergence from anesthesia.

\section{Nitrous oxide $\left(\mathrm{N}_{2} \mathrm{O}\right)$}

$\mathrm{N}_{2} \mathrm{O}$ is widely used in anesthesia because of its amnestic and analgesic properties, as well as its ability to reduce the requirements of expensive inhaled and iv anesthetic drugs. However, the use of $\mathrm{N}_{2} \mathrm{O}$ during laparoscopic procedures remains controversial because of concerns regarding its ability to diffuse into the bowel lumen, causing distention and impaired surgical access. However, Taylor et al. ${ }^{12}$ could not find any difference in surgical conditions during laparoscopic cholecystectomy lasting 80 to $90 \mathrm{~min}$ with or without the use of $\mathrm{N}_{2} \mathrm{O}$. Importantly, bowel distention did not increase with time and the surgeons could not differentiate the patients in whom $\mathrm{N}_{2} \mathrm{O}$ was used from those in whom $\mathrm{N}_{2} \mathrm{O}$ was not used.

Use of $\mathrm{N}_{2} \mathrm{O}$ has also been reported to increase the incidence of postoperative nausea and vomiting (PONV). However, a meta-analysis of randomized controlled trials found that the emetic effect of $\mathrm{N}_{2} \mathrm{O}$ is not significant and omitting $\mathrm{N}_{2} \mathrm{O}$ may increase the risk of awareness. ${ }^{13} \mathrm{~A}$ recent large study reported that omission of $\mathrm{N}_{2} \mathrm{O}$ from a propofol-based anesthetic does not increase postoperative adverse events or the time to home readiness. ${ }^{14}$ Thus, there is no convincing reason to avoid $\mathrm{N}_{2} \mathrm{O}$ during laparoscopic procedures. However, omitting $\mathrm{N}_{2} \mathrm{O}$ from the anesthesia regimen may be an option in patients at risk or when there are surgical difficulties.

\section{Local and regional anesthesia}

Shorter laparoscopic procedures such as diagnostic laparoscopy, pain mapping, laparoscopy for infertility and tubal ligation can be performed under local or regional anesthesia. ${ }^{15}$ These procedures are increasingly performed in the office setting. Regional anesthesia has numerous advantages such as early recovery, reduced PONV, lower postoperative pain, and shorter hospital stay. ${ }^{16}$ However, it is necessary to use lower IAP and reduce the degree of head-down tilt. Although laparoscopy in the awake patient appears to be tolerated well, shoulder pain may be a significant intraoperative problem, which may be reduced by producing pneumoperitoneum with $\mathrm{N}_{2} \mathrm{O}$ rather than $\mathrm{CO}_{2} \cdot{ }^{2,15}$ Of note, $\mathrm{N}_{2} \mathrm{O}$ supports combustion and may increase the risk of fire and explosion when used with electrocautery.

\section{PONV}

PONV remains an important concern for surgical patients, as it is extremely unpleasant. PONV is a common complication for both inpatients and outpatients undergoing laparoscopic surgery, regardless of the anesthetic technique used. However, the risks of PONV associated with total iv anesthesia (i.e., propofol-based anesthetic) appear to lower than that associated with inhalation anesthesia. ${ }^{17}$ Recent data suggest that combinations of antiemetics administered prophylatically are more effective than either antiemetic administered alone, particularly in high-risk patients. ${ }^{2}$ A multimodal approach to prevention of PONV includes use of combinations of droperidol $0.625-1 \mathrm{mg}, 5-\mathrm{HT}_{3}$ antagonists (ondansetron $4 \mathrm{mg}$ or dolasetron $12.5-5 \mathrm{mg}$ ), and dexamethasone 4-8 $\mathrm{mg}$, as well as aggressive hydration, use of minimal doses of opioids, and aggressive pain control. ${ }^{2}$

\section{Pain management}

While less intense and less prolonged compared to open operations, pain after laparoscopic surgical procedures may be quite severe, particularly in the early postoperative period. ${ }^{18}$ There is more visceral pain after laparoscopic procedures compared with parietal (i.e., abdominal wall) pain after open abdominal pro- 
cedures. In addition, shoulder pain secondary to diaphragmatic irritation is a frequent occurrence after laparoscopy and can persist for as long as four days. There is a strong correlation between the severity of shoulder pain and the volume of residual subdiaphragmatic gas. ${ }^{2,18}$ Therefore, every attempt should be made to remove as much $\mathrm{CO}_{2}$ as possible at the end of the procedure. The severity of postoperative pain has also been shown to be associated with the duration of surgery. In addition, stretching of the intra-abdominal cavity from higher insufflation pressures significantly increases the severity of pain.

There is increasing evidence suggesting multimodal analgesia techniques [i.e., combination of opioids, nonsteroidal anti-inflammatory drugs (NSAIDs), and local anesthetics] provide more effective pain relief with fewer side effects and earlier recovery. ${ }^{18}$ Infiltration of the laparoscopy portals with a local anesthetic provides excellent analgesia that may outlast the duration of action of the local anesthetic. However, it is important that the local anesthetic is administered not only subcutaneously but also into the subfascial layers. Another simple and effective method of reducing the intensity of postlaparoscopic pain is intraperitoneal instillation of $20 \mathrm{~mL}$ of $0.25 \%$ bupivacaine between the liver and diaphragm.

NSAIDs are now widely used in the perioperative period because of their opioid-sparing effects. Ketorolac administered intravenously in the early intraoperative period reduces intraoperative opioid requirements, postoperative pain scores and analgesic requirements. NSAIDs also appear to be effective when administered orally prior to surgery.

\section{Complications during laparoscopy}

Awareness of the potential complications associated with laparoscopic procedures should allow early detection and treatment, and improve patient care and safety. ${ }^{3}$ The complications associated with laparoscopy include those related to surgical instrumentation, creation of the pneumoperitoneum, and patient positioning.

\section{Complications from surgical instrumentation}

The initial access into the peritoneal cavity can be achieved using the Veress needle that is placed through a subumbilical incision. Because the Veress needle is placed blindly, there is a potential for its misplacement in the subcutaneous space, vascular space, viscus, omentum, mesentery, or retroperitoneum. Uncontrollable hemorrhage from injury to major vessels caused by surgical instrumentation can cause significant hypotension. On the other hand, concealed bleeding from vessel injury can present in the postop- erative period as a fall in hematocrit values. Stomach injuries can be reduced by gastric decompression prior to placement of the Veress needle. Similarly, decompression of the urinary bladder by placement of a urinary catheter or asking patients to void prior to surgery should decrease the possibility of bladder trauma. The complications associated with the Veress needle may be avoided by placement of the first trocar through a minilaparotomy incision. ${ }^{3}$

\section{Cardiovascular complications}

Although rare, acute cardiovascular collapse during laparoscopy has been reported and may be caused by profound vasovagal reaction, cardiac dysrhythmias, acute blood loss, myocardial dysfunction, tension pneumothorax, venous gas embolism, severe respiratory acidosis, cardiac tamponade, excessive IAP, and anesthetic drugs. In addition to routine evaluation (i.e., depth of anesthesia and intravascular volume status), it must be confirmed that the IAP have not exceeded $15 \mathrm{mmHg}$. If these measures are inadequate, deflation of the abdomen may be needed. In patients with a significant fall in cardiac output and high systemic vascular resistance, pharmacologic interventions with nitroglycerin and/or $\beta$-adrenergic agonists may be necessary to maintain hemodynamic status. ${ }^{4}$ Rarely, it may be necessary to convert to an "open" procedure if the significant impairment persists.

\section{Pulmonary complications}

The pulmonary changes during laparoscopy can cause severe hypoxemia and hypercarbia. The differential diagnosis of significant pulmonary dysfunction includes $\mathrm{CO}_{2}$ absorption, hypoventilation (airway obstruction, ventilator or breathing circuit leaks), increased dead space (associated with abdominal distention, position of the patient, mechanical ventilation), endobronchial intubation, reduced cardiac output, $\mathrm{CO}_{2}$ embolism, pneumothorax, pneumomediastinum, pneumopericardium, and subcutaneous emphysema.

\section{Subcutaneous emphysema}

Subcutaneous emphysema can occur from inadvertent extraperitoneal insufflation or from extension of extraperitoneal insufflation. Subcutaneous emphysema increases the area for $\mathrm{CO}_{2}$ diffusion, which can result in significant hypercarbia and respiratory acidosis. It can be diagnosed by the development of crepitus and increase in end-tidal $\mathrm{CO}_{2}$ concentrations. In most cases, subcutaneous emphysema resolves soon after abdominal deflation. However, in some patients it may be necessary to deflate the abdomen to allow $\mathrm{CO}_{2}$ elimination. If the emphysema extends to the chest wall 
and the neck, the $\mathrm{CO}_{2}$ can track to the thorax and mediastinum, thereby resulting in pneumothorax or pneumomediastinum. Therefore, a chest $x$-ray should be obtained if a patient develops cervical emphysema.

\section{Pneumothorax}

Pneumothorax can occur from insufflated $\mathrm{CO}_{2}$ tracking into the thorax through a tear in the visceral peritoneum, breach of the parietal pleura during dissection around the esophagus, or a congenital defect in the diaphragm. ${ }^{19,20}$ Early diagnosis and treatment of pneumothorax can be achieved by maintaining a high index of suspicion. It can be diagnosed by the development of increased peak airway pressures, reduced oxygen saturation, and in rare cases significant hypotension and cardiac arrest. Treatment of pneumothorax includes deflation of the abdomen and supportive treatment. If there is minimal physiologic compromise conservative treatment with close observation may be adequate because $\mathrm{CO}_{2}$ is rapidly absorbed. In patients with severe compromise, placement of an intercostal cannula may be necessary, followed by a chest drain if reaccumulation occurs. Conversion to an open procedure might be necessary after stabilization.

\section{Hypothermia}

It is generally expected that the degree of hypothermia during a closed procedure such as laparoscopy would be less than that during an open procedure because the abdominal contents are not exposed to the atmosphere. However, the incidence of hypothermia during laparoscopic procedures is similar to that of open abdominal operations. ${ }^{21,22}$ Although built-in heating elements to warm the insufflating gas have become available commercially, warming (without humidification) of insufflation gas does not prevent hypothermia and is thus unnecessary during laparoscopy. Some authors have reported that the use of heated humidified insufflating gas may reduce postoperative morbidity, including postoperative pain. However, larger studies are necessary to show the advantages of humidifying insufflating gas. Prevention of surgical hypothermia should address other, more significant, sources of heat loss. It is important to take an aggressive approach in maintaining patients' temperatures including humidification of anesthetic gases, infusion of heated iv fluids, prewarming irrigant fluids, and forced-air warming to improve postoperative outcome.

\section{Postoperative complications}

After deflation of the abdomen, it may require approximately $45 \mathrm{~min}$ for the arterial $\mathrm{CO}_{2}$ concentrations to return to preinsufflation values. Of note, normaliza- tion of $\mathrm{CO}_{2}$ concentration may take longer after extraperitoneal insufflation than that after intraperitoneal insufflation. ${ }^{23}$ Impaired postoperative ventilation from residual anesthetics and/or neuromuscular blockade may result in significant hypercapnia. In patients with significant respiratory dysfunction and restricted $\mathrm{CO}_{2}$ clearance, positive pressure ventilation may be required in the postoperative period until the patient can eliminate the $\mathrm{CO}_{2}$ load with resumption of spontaneous respiration.

\section{Summary}

With improvement in technology and growing surgical expertise, more extensive and prolonged laparoscopic procedures will be performed in a wide range of patients. Modification of the anesthetic technique and the prevention of common postoperative complications including pain, nausea, and vomiting using the multimodal approach should allow early recovery. Although the laparoscopic approach provides significant benefits, a thorough understanding of the associated cardiopulmonary changes and the potential complications is necessary to maintain patient safety.

\section{References}

1 Smith I. Anesthesia for laparoscopy with emphasis on outpatient laparoscopy. Anesthesiol Clin North America 2001; 19: 21-41.

2 O'Malley C, Cunningham AJ. Physiologic changes during laparoscopy. Anesthesiol Clin North America 2001; 19: 1-19.

3 Joshi GP. Complications of laparoscopic surgery. Anesthesiol Clin North America 2001; 19: 89-105.

4 Hein HAT, Joshi GP, Ramsay MAE, et al. Hemodynamic changes during laparoscopic cholecystectomy in patients with severe cardiopulmonary disease. J Clin Anesth 1997; 9: 261-5.

5 Gannedabl P, Odelberg S, Brodin LA, Sollevi A. Effects of posture and pneumoperitoneum during anesthesia on the indices of left ventricular filling. Acta Anaesthesiol Scand 1996; 40: 160-6.

6 Ishizaki $\Upsilon$, Bandai $\Upsilon$, Shimomura K, Abe H, Obtomo $\Upsilon$, Idezuki $\Upsilon$. Safe intra-abdominal pressure of $\mathrm{CO}_{2}$ pneumoperitoneum during laparoscopic surgery. Surgery 1993; 114: 549-4.

7 Dexter SP, Vucevic M, Gibson J, McMahon MJ. Hemodynamic consequences of high- and low-pressure capnoperitoneum during laparoscopic cholecystectomy. Surg Endosc 1999; 9: 376-81.

8 Talamini MA, Mendoza-Sagaon M, Gitzelmann CA, et al. Increased mediastinal pressure and decreased cardiac output during laparoscopic Nissen fundoplication. Surgery $1997 ; 122$ : 345-52. 
9 Joshi GP. Fast tracking in Outpatient Surgery. Curr Opin Anaesthesiol 2001; 14: 635-9.

10 Song D, Joshi GP, White PF. Fast-track eligibility after ambulatory anesthesia: a comparison of desflurane, sevoflurane, and propofol. Anesth Analg 1998; 86: 267-73.

11 Song D, Joshi GP, White PF. Titration of volatile anesthetics using bispectral index facilitates recovery after ambulatory anesthesia. Anesthesiology 1997; 87: 842-8.

12 Taylor E, Feinstein R, White PF, Soper N. Anesthesia for laparoscopic cholecystectomy: is nitrous oxide contraindicated? Anesthesiology 1992; 76: 541-3.

13 Tramer M, Moore A, McQuay H. Omitting nitrous oxide in general anesthesia: meta-analysis of intraoperative awareness and postoperative emesis in randomized controlled trials. Br J Anaesth 1996; 76: 186-93.

14 Arellano RJ, Pole ML, Rafuse SE, et al. Omission of nitrous oxide from a propofol-based anesthetic does not affect the recovery of women undergoing outpatient gynecologic surgery. Anesthesiology 2000; 93: 332-9.

15 Collins LM, Vaghadia H. Regional anesthesia for laparoscopy. Anesthesiol Clin North America 2001; 19: 43-56.

16 Joshi GP. Recent developments in regional anesthesia for ambulatory surgery. Curr Opin Anaesthesiol 1999; 12: 643-7.

17 Visser K, Hassink EA, Bonsel GJ, Maon J, Kalkman CJ. Randomized controlled trail of total intravenous anesthesia with propofol versus inhalation anesthesia with isoflurane-nitrous oxide. Postoperative nausea and vomiting and economic analysis. Anesthesiology 2001; 95: 616-26.

18 Joshi GP. Pain management after ambulatory surgery. Ambulatory Surgery 1999; 7: 3-12.

19 Yee R, Hyde PR, Currie JS. Pneumothorax during laparoscopic Nissen fundoplication. Anaesth Intensive Care 1996; 24: 93-6.

20 Dawson R, Fergusson CJ. Life-threatening tension pneumothorax during laparoscopic Cholecystectomy. Surg Laparosc Endosc 1997; 7: 271-2.

21 Makinen $M-T$. Comparison of body temperature changes during laparoscopic and open cholecystectomy. Acta Anaesthesiol Scand 1997; 41: 736-40.

22 Stewart BT, Stitz RW, Tuch MM, Lumley JW. Hypothermia in open and laparoscopic colorectal surgery. Dis Colon Rectum 1999; 42: 1292-5.

23 Mullet CE, Viale JP, Sagnard PE, et al. Pulmonary $\mathrm{CO}_{2}$ elimination during surgical procedures using intra- or extraperitoneal $\mathrm{CO}_{2}$ insufflation. Anesth Analg 1993; 76: 622-6. 